\title{
Antibody mediated protection of immunized macaques against pathogenic SHIV challenge
}

\author{
Indresh K Srivastava*1, Elaine Kan ${ }^{1}$, Yide Sun ${ }^{1}$, Harold Legg ${ }^{1}$, Queency Lee ${ }^{1}$, \\ Brian Burke ${ }^{1}$, Catherine Greer ${ }^{1}$, Ying Lian ${ }^{1}$, Leonidas Stamatatos ${ }^{2}$, \\ Jeffrey B Ulmer ${ }^{1}$, Michael Vajdy ${ }^{1}$, John Donnelly ${ }^{1}$, Chris Miller ${ }^{3}$ and \\ Susan W Barnett ${ }^{1}$
}

Address: ${ }^{1}$ Novartis Vaccines and Diagnostic, 4560 Horton Street Emeryville, California, USA, ${ }^{2}$ Department of Pathobiology, Seattle Biomedical Research Institute, Seattle, Washington, USA and ${ }^{3}$ California Regional Primate Center, UC Davis, Davis California, USA

* Corresponding author

from 2006 International Meeting of The Institute of Human Virology Baltimore, USA. 17-2I November, 2006

Published: 21 December 2006

Retrovirology 2006, 3(SuppI I):S27 doi:I0.I186/1742-4690-3-SI-S27

(c) 2006 Srivastava et al; licensee BioMed Central Ltd.

The primary objective of our current HIV vaccine research program is to evaluate novel alphavirus-based and Envelope (Env) protein-based vaccine formulations either alone or in prime boost regimen for improved immunogenicity and efficacy over other HIV vaccine approaches. The outcome of this work will be the production of candidate vaccine components that can be further advanced to clinical trials in human subjects. For these studies we employ viral genes derived from SF162 a primary subtype B HIV-1 isolate in alphavirus replicon particle-based vaccines. A chimeric alphavirus vector system (VEE/SIN) is used that is composed of the Venezuelan Equine Encephalitis (VEE) replicon combined with the Sindbis virus structural proteins. We have performed a proof of concept study where macaques were primed with VEE/SIN, boosted with o-gp140DV2SF162, and challenged intravenously by pathogenic SHIVSF162P4. In the next study we evaluated these recombinant VEE/SIN vaccines alone, and also in combination with DV2-trimer derived from SF162 for their ability to protect against mucosal SHIVSF162P4 challenge. Both parenteral and mucosal routes of immunization have been evaluated to identify the optimal regimen(s) for the induction of potent and protective humoral and cellular responses in rhesus macaques. In the third study, we evaluated the ability of DV2SF162 trimer to protect against against mucosal SHIVSF162P4 challenge. Both parenteral and mucosal routes of immunization have been evaluated. Results so far indicate that the recombinant VEE/SIN particles induced antigen-specific CD4+ and CD8+ T cell responses as well as neutralizing antibodies in small animals (mice and rabbits) and non-human primates. Moreover, intramuscular immunization with VEE/SIN particles encoding SIV gag or gagpol and HIV env is quite effective in priming for potent antiEnv antibody responses. However, it seems intramuscular boosting of alpha primed animals with ogp140DV2SF162 in MF59 is critical for blocking and/or reducing acute phase viremia following either intravenous or intrarectal SHIVSF162P4 challenges of rhesus macaques. Furthermore, macaques that were immunized intranasally with o-gp140DV2SF162 protein in the LTK63 mucosal adjuvant followed by intramuscular immunization with o-gp140DV2SF162 in MF59 were also protected against SHIVSF162P4 challenge. Protection in these studies appears to occur after the induction of high titer serum neutralizing antibodies that recognize the challenge virus. 